\title{
DISTANCE AND BOUNDARIES ISSUES IN THE TRANSITION FROM FACE-TO-FACE TALKING THERAPY TO ONLINE THERAPY IN THE TIME OF COVID-19
}

\author{
VERONICA MARIA MATEESCU1
}

\begin{abstract}
The primary main aim of this article is to explore the changes and adjustments brought about by the COVID-19 pandemic to the therapeutic process in the transition from face-to-face talking therapy to online therapy. Online therapy sessions, once a niche, have now become the norm in therapeutic work. This, more than ever before, raises the question of the efficacy of online therapy compared to face-to-face talking therapy. A related question is how the "classical" elements of in-person therapies (especially the psychodynamic, affective and relational-based), such as: the therapeutic alliance, the therapeutic containing space, the therapeutic relationship, etc. work in the on-line setting. This article draws on a primary qualitative exploratory research carried among Romanian clients who undergo a psychodynamic type of therapy and who transitioned from face-to-face to online therapy as a reaction to the new constraints engendered by the COVID-19 pandemic and after the measures were relaxed, the transition from on-line to in-person therapy. Our focus is on how they experience online therapy compared to face-to-face therapy in terms of intimacy, therapeutic frame and efficacy, as well as on the boundaries challenged, erased and created by the switch between the two types of therapy settings.
\end{abstract}

Keywords: online therapy; therapeutic boundaries; space and time boundaries; work-life balance; intimacy boundaries

\section{Introduction}

"Therapy, whatever its school or approach, was always to some degree teletherapy, the crossing of distances." (Peters in: Zeavin, 2021: xi). Teletherapy or e-therapy/online therapy/video-therapy is a form of telehealth which uses high-resolution, live video conferencing in the process of psychological treatment

1 Department of Sociology, Faculty of Sociology and Social Work, Babeș-Bolyai University, Cluj-Napoca. Email: veronica.mateescu@ubbcluj.ro 
(Goode and Shinkle, 2019). The COVID-19 pandemic forced both clients and therapists, regardless of their training, certification and experience, to switch from in-person therapy to online therapy (Mental Health America, 2021). One of the questions raised is the efficacy of online therapy in the long run and for every type of problem. A meta-analysis conducted by Mental Health America (2021) on the recent studies on teletherapy in the time of COVID-19 indicates that teletherapy is a useful therapeutic tool for the short-term and for emergencies like COVID-19, but that it shouldn't permanently replace in-person therapy. Another conclusion is that the therapeutic full presence, a necessary element for an effective therapeutic relationship, is compromised in the virtual setting, which does not provide the possibility to create an emotional connection and to build trust (Mental Health America, 2021).

The space, place, time, relationship, intimacy and personhood are the main areas impacted by the switch from face-to-face talking therapy to online therapy (Mateescu, 2020). The question at stake is the efficacy of the therapeutic process in the online setting and how the therapeutic relationship and/or the therapeutic techniques can contribute to it. A related question is the online therapy's efficacy and suitability, depending on the therapists, on the clients' problems and the type of therapy (e.g. cognitive and behavioural therapies that are more technique-based, as opposed to affect-based and relational therapies) (Weinberg and Rolnick, 2020).

The switch from face-to-face talking therapy to e-therapy has hardened the therapist's ability to create a safe and containing space. The therapeutic space is regarded as one of the most important elements of the dynamic psychotherapy setting. It goes beyond the physical space and includes a series of elements such as intimacy, confidentiality, duration, time, frequency, fees, breaks, cancellations, etc. which all together contribute to the creation of a secure transitional space (Winnicott, 2005) and set the terms of the ongoing therapeutic relationship (Weinberg and Rolnick, 2020). In online therapy, the physical therapeutic presence, which is regarded as a critical element in therapy efficacy, (Geller and Greenberg, 2002) needs to be rethought. Along with these elements, the Self is negatively affected in online therapy, as it becomes a "disembodied self" (Weinberg and Rolnick, 2020). Conversely, Lemma (2017) argues for an embodied presence/self in online, mediated therapy, the difference lying only in the way we perceive and experience it. The individuals develop "internal models of space" (Dix, 2009) resulting from a mix between subjective and objective experiences, between real and imagined spatiality (Doyle, 2017). Thus, the online space becomes not only a metaphor, but a mix of concrete and virtual elements, constructed by individuals through cognitive and affective elements which allow them the "telepresence" (Berger, 2020). Lemma (2017) raises the 
issues of "simulating presence", resulting from the alteration and mediation of intimacy by technology. Agar (2020) talks about a new kind of intimacy: the online/e-intimacy, which has similar traits to that of face-to-face therapeutic large groups such as a feeling of belonging and the creation of a cohesive climate.

Also, intimacy can be created through non-verbal communication, because even if the body-to-body communication misses, there is still emotional communication and body sensations in the online therapy (Weinberg and Rolnick, 2020). The therapist is required to focus on those visible non-verbal cues (i.e. eyes, posture, etc.) and on active listening. Some therapists impose distancing and seating rules in order to replicate the face-to-face physical space, but the fidelity of replication and the therapeutic effects are debatable (Weinberg and Rolnick, 2020).

All these elements build the setting for the therapeutic relationship. Other important aspects are also involved, such as the creation of the therapeutic alliance, the transference and countertransference processes, detachment and the end of therapy, which are all altered by the switch from face-to-face to online therapy.

\section{Distance and boundaries in online therapy}

The main boundaries challenged, created or erased by the switch from face-toface talking therapy to online therapy are: therapeutic boundaries; intimacy boundaries for both the therapist and the client; space and time boundaries; social class and income boundaries. (Mateescu, 2020: 74)

The therapeutic boundaries are closely linked to intimacy, space, and time boundaries. They are the most challenged in online therapy, compared to face-to-face therapy (Drum and Littleton, 2014). Therapeutic boundaries and the way they are created and maintained are extremely important for the efficacy of therapeutic processes. Spatial boundaries allow for the creation of a secure, predictable, confidential and trustworthy space, of a personal, transitional space (Punzi and Singer, 2018). The transitional space is a mixture of external realities/boundaries and individual/shared phantasies (Punzi and Singer, 2018). In online therapy, the client/patient becomes a co-constructor of the transitional space in equal measure with the therapist (Mateescu, 2020). A study on teletherapy during COVID-19 and the transitioning from face-to-face talking therapy to online therapy (Lokai et al., 2021) revealed a shift in the therapist's role, who was "normalised" by the patient and seen "more as a person". The therapists also indicated changes in the therapeutic relationship and in their role. For example, in one case, a therapist shared his therapeutic process notes with the 
client, something that was totally excluded in face-to-face therapy. In some other cases, the therapist felt that his role had changed from therapist to a coach and problem-solver (Lokai et al., 2021).

Spatial boundaries are closely linked to intimacy boundaries for both the therapist and the client. The already mentioned study of Lokai et al. (2021) revealed an increase in the therapist's self-disclosure and the loosening of therapeutic boundaries in the transitioning from in-person therapy to online therapy. An analysis made by Zur, Williams, Lehavot (2009) about the effects that the Internet technology had on the classical therapeutic instruments of intentional self-disclosure and transparency also indicates a major change in the direction of their increasing and the lack of control of the therapist over it. The therapists can also get more information about their clients, and the change of the therapeutic frame offers direct and indirect information about the client's life and enhances the unintentional self-disclosure (Mateescu, 2020).

Another important change brought about by the online therapy is to the geographical space and time boundaries. Thus, clients from remote locations or from areas that lack therapy services can still benefit from therapy. It is also the case of those who are concerned with confidentiality issues that can be encountered in small communities, where there is a great overlap between social, personal, business and professional relationships (Alyami, 2015) and an informal informationsharing network (Helbok, 2003). Additionally, the online therapy provides expat clients with the opportunity to continue or to have therapy in their native language. It is an important benefit for the therapeutic alliance and the efficacy of the therapeutic process (Curtis, 2017), because the native language is more emotionrelated, being learned in the primary socialization and it is an important factor in the development of an individual's identity (Caldwell-Harris, 2014).

The social class and income boundaries were also challenged by the online therapy. The underserved groups, such as low-income individuals, now have better access because there are no extra-costs (e.g.: transportation). Also, the social class differences are toned-down due to easier access to technology and an Internet connection (Liang et al., 2003). Another undeniable benefit is better time management and consequently, a better work-life balance (Lemma, 2017).

\section{Distance and boundaries issues in therapy in the transition from face-to-face talking therapy to online therapy in the time of COVID-19}

The analysis of distance and boundaries issues in therapy in the transition from in-person therapy to online therapy draws on exploratory qualitative research. The data collection method is that of an in-depth unstructured interview. Each participant was interviewed over the online video platforms Skype and 
Whatsapp and face-to-face, for a duration ranging between 45 minutes and 60 minutes. The participants are ten clients who are undergoing psychodynamic therapy. All participants experienced both in-person and on-line therapy. Nine of them experienced a transition from face-to-face talking therapy to online therapy as a reaction to the new constraints engendered by the COVID-19 pandemic. One of them began the therapy online during the COVID-19 pandemic and then switched to in-person therapy, when the therapist re-started to work face-to-face. The participants' therapeutic processes are ongoing. They had on-line therapy for 6 months up to 1 year and 9 months and in-person therapy between 4 months and 3 years. Seven of them came back to in-person therapy when the therapist started to work face-to-face again, three of them continued to have therapies on-line.

\section{The therapeutic space boundaries}

In online therapy, the client becomes the co-creator of the therapeutic space. A common theme is the concern to create a space for therapy, in order to have intimacy and to separate it from the daily routine. "I moved into the living room, I needed the space to be clean, airy, and neutral. I needed to fill the space, not to be filled by it." (G.G.) Another common theme is the difficulty to connect with the therapeutic processes because there was no break between other activities or working schedules and the therapy sessions, they were a continuum. Those who had a previous face-to-face therapy experience added elements that helped them to recreate the atmosphere of the secure physical space, such as having a pack of tissues during the online sessions. "Before starting a therapy session, I prepare to have some tissues at hand. I knew that at the practice there were some on the table, so I also put them on the table." (A.R.). Also, they paid attention to the lighting, to the background, the sitting places, the phone position, the quality of the image and of the sound.

Another common theme is the tendency to perform, the lack of authenticity and the feeling of unnaturalness.

The first (online) sessions were strange, there was a feeling of insecurity, I wasn't sure that I could talk about everything that bothered me, it was like I was performing in front of an audience assisted by a public. After a while, I got used to it, but not one online session was $100 \%$ as comfortable as a face-to-face session. (C.A.)

When I do therapy in person, I leave a part of myself, I don't analyse myself so much, how I look and how other people see me. Online, it is like I film myself. It's more than mirroring, it is as if I recorded myself. And by using parts of myself, which is very personal, and combining them with technology is like I had to present my trauma on the news, it is like a disconnection. It feels like being part of a theatre play, a fictitious therapy session that is semi-real. (F.M.) 


\section{Intimacy}

A common theme is the concern for having intimacy, which was difficult for those who do not live alone and even in those cases there was a concern for confidentiality, a fear that the conversation might be witnessed by the neighbours.

The feeling of intimacy diminished. I had a strange feeling. I didn't feel the therapist was a strange person, because I already knew her, but I could feel a distance. (...) Many times I felt lonely, I was perfectly aware of the distance and of the fact that we weren't in the same room. At the practice, the rooms are small, cozy, perfect for the therapy, at home I have a big room, a living room, where there is an echo. The face-to-face therapeutic setting offers me much more safety, intimacy and there is a feeling of coziness. Maybe because I am a person who likes to be around other people, to feel their physical presence; pandemic or not, I used to like face-to-face meetings, I prefer the physical presence. I feel that I have a greater freedom to express my thoughts, I am not focused on a single point, which is the phone. It becomes an extra-worry. (Online therapy) is like having therapy by looking through the keyhole. (C.A.)

\section{The therapeutic relationship}

The therapeutic relationship wasn't negatively impacted by the transition from in-person to online therapy. In some cases, it was in fact reinforced, because it was seen as a secure continuity in a changing and insecure reality. Coming back to in-person therapy was described as a very happy and liberating process, as a sign of "normalisation" of life and a way to reconnect.

A recurring theme is that of a feeling of distance in online therapy, despite a good therapeutic relationship, while in face-to-face therapy the therapist is perceived as more able to "read", to "observe" and "to put pressure".

I was happy to come back face-to-face. My body needed grounding, I needed to be pulled out of my spaces and to be exposed in this therapeutic relationship. (...) face-to-face I have the feeling that the therapist reads me better and can catch me more easily. (D.D.)

When you are face-to-face, you are somehow 'forced' to be there. Online sessions are more sugar coated, you can make the therapy be less tough, less cruel. Online therapy is like a story in a frame. (F.M.)

Another recurring theme in how people describe their online therapy experience is that of a greater possibility to avoid uncomfortable topics as compared to face-to-face sessions. The defence mechanisms are strengthened by the screen-mediated communication and by the intimacy of the personal space. 
At the practice, I got used to talking, it is a space dedicated to therapy alone, there is a room only for that. Here, in my space, there is a lot of stuff. (At the practice), after I finish my therapy, I go on living my life, nobody knows what I said in there, it stays there. Here (online), I had the impression that I could avoid certain topics easier, there I feel somehow cornered, like I have to give an answer, even if I say that I don't know. It is easier, the therapist doesn't see me so well, I can tap my feet, I can look somewhere else. At the practice, the therapist has control, even if I believe I have it. That is her space, not mine. (M.M)

Conversely, in some cases the intimacy of the personal space favoured the openness to address difficult topics and show the vulnerabilities.

(Online) It felt relaxing from the perspective of space intimacy. I felt more free, it is a space where I have the most intimate relationship with myself. Maybe it is more comfortable for a person who withdraws in her own space to be pulled out of it, to take a shower, to take a cab and to be pulled out of the suffering. At the same time, it is the space where I withdraw myself and it is like letting the doctor come to my place. (D.D.)

\section{The question of the Self}

A common theme is the body as a partial object, as a result of the screen mediation. In some cases, the fact that the clients could see their image and parts of their body on the screen had a negative effect on the therapeutic process, because it amplified previous body issues or because they could avoid and neglect bigger body issues (eg.: weight problems).

You can see yourself on the screen. It is like a reminder. I look at my therapist a lot. Before I didn't look at myself, because I didn't like myself, but in fact, out of the corner of your eye you can see yourself. I can see myself up from my shoulders and that produces a disconnection from my body. I don't see myself entirely, I realised that hasn't only been happening in therapy, but also in the 2 years since I have been working on Zoom. As for my body issues, I no longer saw them, my body wasn't mine, it didn't belong to me. Now, I feel better and I have begun paying attention to it. (G.G.)

In other cases, it had a positive effect, because it gave the client the opportunity to self-observe, better understand the emotions and be more aware. 
I didn't like seeing my face. But, in a way, it was helpful, because I could see what I look like when I laugh, when I cry, when I don't know how to answer a question. For instance, I noticed I had a tic and now I try to get rid of it. I became more aware of myself. (A.A.)

There is a lack of visual cues in online therapy, from both the client and the therapist, that can compromise the perception and the interpretation on both parts. Also, it allows the client to better hide the emotional reactions to uncomfortable topics.

(Online) there is a kind of securitization that can easily become a shield. There is a thin line. It can be a way of protection. It is important if you see someone's hand or not, if he/she taps her feet or not. It is easier to hide and you are tempted to do it. That's why for many people it is more comfortable to stay online. It is not only a question of time. (Online therapy) It helps you avoid difficult topics, it is somehow perverted, because you don't go to the practice, where you have to expose yourself. (B.B.)

\section{Time boundaries}

In terms of time management, online therapy is preferred to face-toface therapy, because it allows more flexibility and continuity.

Online, you finish faster and you can go back to your business, but it is debatable if that is good or bad for therapy. I like a hybrid system (online and face-to-face), because I don't have to be stuck, I can go on vacation or have leisure time and also have therapy. But I see it as a plan B. (C.A.)

Online therapy is a good option in terms of time management, you can have your sessions almost any time after your work schedule, you can go on vacation and to take one hour for therapy. (A.R.)

It is comfortable to know that, if need be, you can shift between face-to-face and online therapy and that there is continuity even if I decide to go away for 6 months. (D.D.)

In terms of therapy efficacy, the time frame allotted to face-to-face therapy is more efficient and represents a significant part of the therapeutic process. There is a common thread in how the interviewed subjects describe the time spent in order to go to the practice as a time of reflection and preparation for therapy and of the time after therapy as a time of reflection, accumulation and soothing. 
Online, you can save time by not traveling to the practice, but, in fact, the time you spend traveling in order to go there and then to go back home is very good, because that is a time for myself. I don't know why I don't allow for this time when I am online, maybe because I shift suddenly from one state of mind to another, the dog is barking, your cat is meowing. There, (face-to-face) it is different, there is a moment of I don't know what, of respite. Even if the therapy session is hard, I return in a good mood. If I didn't drive there by car it would be better. (M.M.)

\section{Conclusion}

The transition from in-person therapy to online therapy didn't impact the therapeutic alliance and relationship negatively, but, in some cases it attenuated the therapy efficacy for reasons of intimacy, lack of concentration on account of squeezing in therapy sessions among other domestic activities and/or work schedules, especially in the case of those who work from home. Another important factor that moderated negatively the therapy efficacy was the screen-mediated interaction, which transformed the bodies into partial objects and distorted the perception and the interpretation of visual cues from both the therapist and the client. Also, in some cases, online therapy was seen as allowing, more than face-to-face therapy, to avoid difficult topics and to hide some emotional reactions. In some other cases, the intimacy of the personal space in online therapy favoured the openness toward sensitive issues, because the clients didn't feel so exposed to the pressure of the therapist and of the physical interaction which formalises the process. Those participants who came back to face-to-face therapy after a period of on-line therapy felt more connected and more free to express themselves in a space that is dedicated only to therapy and assessed the non-verbal communication as better than in online therapy/sessions, even if at the practice both the therapist and the clients have to wear masks nowadays. Another important element that was regained and has been considered an added value to it is the time frame allotted in order to go to therapy, to undergo therapy and to come back, a time frame that is an important part of the therapeutic process and contributes to its efficacy. Also, there are some small rituals at the practice (i.e: having a cup of coffee or tea, sitting in the waiting room, etc.) before the therapy begins which are part of the therapeutic relationship and space and constitute a part of the therapeutic process itself.

Those participants who decided to continue the therapy online made this choice for reasons of time management. They consider the on-line therapy as effective as the face-to-face therapy, with a small disclaimer, that it lacks the energy and the connection of meeting in person. 
All participants, regardless whether they prefer face-to-face or inperson therapy, consider that a hybrid system is workable and allows for more flexibility and continuity.

The transition from face-to-face therapy to online therapy erased some of the boundaries raised by face-to-face therapy in terms of flexibility, mobility, geographical distance, time management, work-life arrangements and efficacy in some types of disorders (Mateescu, 2020). At the same time, it creates some others, because of its perceived distance, poor visual cues, the body becoming a partial object, the lack of intimacy and the fatigue provoked by its insertion in the continuum of online activities that working from home entails, which have all become the norm in many types of jobs.

\section{REFERENCES}

Agar, Gily (2020). The Clinic Offers No Advantage Over the Screen, for Relationship is Everything. In Weinberg, Haim and Rolnick, Arnon (eds.). Theory and Practice of Online Therapy. Internet-delivered Interventions for Individuals, Groups, Families, and Organisation. NY \& London: Routledge, pp. 66-78.

Alyami, Mohsen (2015). Ethical Dilemmas of Confidentiality in Rural Communities. Management and Recommendations. Arab Journal of Psy Science, 46: 129-133

Berger, Viktor (13 May 2020). Phenomenology of Online Spaces: Interpreting Late Modern Spatialities. Human Studies, [https://www.researchgate.net/publication/341345177], 20 June 2020.

Dix, Alan (2009). Paths and Patches: Patterns of Geognosy and Gnosis. In Turner Phil; Turner, Susan; and Davenport, Elisabeth (eds.), Exploration of space, technology, and spatiality: interdisciplinary perspectives. NY: Information Science Reference: Hershey, 1-16.

Doyle, Denise (2017). Avatar Lives: Narratives of Transformation and Identity. In Gackenbach, Jayne and Bown, Johnathan (eds.). Boundaries of Self and Reality Online: Implications of Digitally Constructed Realities. 1st Edition. London, UK: Academic Press, 57-74.

Drum, Katherine B. and Littleton, Heather L. (2014). Therapeutic boundaries in telepsychology: Unique issues and best practice recommendations. Professional Psychology Research and Practice, 45(3); 309-315.

Geller, Shari M. and Greenberg, Leslie S. (December 2002). Therapeutic Presence: Therapists' experience of presence in the psychotherapy encounter. PersonCentered and Experiential Psychotherapies, 1 (1-2): 72-73.

Goode, Howard and Shinkle, Elana (2019). What is Teletherapy? A Helpful and Definitive Guide. Retrieved from: https://globalteletherapy.com/what-isteletherapy/,12/22/2021 
Helbok Craig M. (2003). The practice of psychology in rural communities: potential ethical dilemmas. Ethics Behav., 13(4): 367-84

Lemma, Alessandra (2017). The Digital Age on the Couch. Psychoanalytic Practice and New Media. London \& NY: Routledge. Taylor \& Francis Group.

Liang Belle; Duffy, Nicole and Commins, Meghan (2003). The Online World, the Internet, Social Class, and Counseling. In William Ming Liu (ed.), The Oxford Handbook of Social Class in Counseling. Oxford University Press: 260-272

Lokai, Andrew D., Chen Liqiaonan, Terry, Kendra, Eubanks, Catherine F., and Muran, Christopher (2021). Transitioning to virtual space: Teletherapy in the time of COVID-19. Psychotherapy Bulletin, 56(2): 29-34.

Mateescu, Veronica Maria (2020). Online Therapy-Short-Term Solution or Opportunity During the Covid-19 Pandemic? An overview of the dynamics of the therapeutic field in Romania. Studia Universitatis Babes-Bolyai Studia Europaea, 65(2): 7189.

Mental Health America (2021). Teletherapy During COVID-19: What The Research Says. Retrieved from: [https://mhanational.org/teletherapy-during-covid-19-whatresearch-says], 12/21/2021

Punzi, Elizabeth and Singer, Cristoph (2018). Any room won't do.' Clinical psychologists' understanding of the consulting room. An interview study. Psychodynamic Practice, 24(4): 319-333.

Weinberg, Haim and Rolnick, Arnon (2020). Introduction. In Weinberg, Haim and Rolnick, Arnon (eds.), Theory and Practice of Online Therapy. Internet-delivered Interventions for Individuals, Groups, Families, and Organization. NY \& London: Routledge, 1-10.

Winnicott, Donald Woods (2005). Playing and Reality. London \& NY: Routledge Classics.

Zeavin, Hannah (2021). The distance cure: a history of teletherapy. Cambridge, Massachusetts: The MIT Press.

Zur, Ofer; Williams, Martin H. and Lehavot Keren; Knapp, Samuel (February 2009). Psychotherapist Self-Disclosure and Transparency in the Internet Age. Professional Psychology Research and Practice, 40 (1): 22-30. 